\title{
Second Variation of Energy for Minimal Surfaces in Riemannian Manifolds
}

\author{
John Douglas Moore \\ Department of Mathematics \\ University of California \\ Santa Barbara, CA, USA 93106 \\ e-mail: moore@math.ucsb.edu
}

\begin{abstract}
This article describes a formula for second variation of energy for twodimensional parametrized minimal surfaces, in which the conformal structure on the two-dimensional domain is allowed to vary. Moreover, it shows that minimal surfaces with branch points have tangential Jacobi fields which are not visible via the standard formulae for second variation of area which is commonly used to study stability of minimal surfaces.
\end{abstract}

\section{Introduction}

The formula for second variation of area is one of the principal tools used to study stability of minimal submanifolds of Riemannian manifolds. However, the second variation formula which is in standard use (see Simons [10], Lawson [4], Micallef [6], Micallef and Wolfson [8], Fraser [3], as well as others) restricts attention to normal variation. This version of the second variation formula has the advantage that it can be extended to more general objects such as integral currents and varifolds. When the domain has two dimensions, however, stronger results are sometimes achievable using a parametrized theory in which closed minimal surfaces are regarded as maps from a compact surface $\Sigma$ into a Riemannian manifold $M$, the maps being allowed to have branch points. In this context, one needs a formula for second variation for the two-variable energy function

$$
E: \operatorname{Map}(\Sigma, M) \times \mathcal{T} \longrightarrow \mathbb{R}
$$

where $\Sigma$ is a closed oriented surface of genus $g$ and $\mathcal{T}$ is the corresponding Teichmüller space.

In this article, we present such a second variation formula for the two-variable energy $E$ (see end of $\S 4$ ). When $E$ is restricted to immersions and variations are normal, the second variation of $E$ differs from the second variation of area only in "divergence terms," and we explain these divergence terms in $\S 5$. The second variation formula shows that if a parametrized minimal surface has branch 
points, these give rise to additional tangential Jacobi fields for $E$ that are not visible when taking only normal variations. The number of such tangential Jacobi fields depend only on the total branching order, and can be calculated via the Riemann-Roch Theorem, as we describe in $\S 7$.

The author thanks the referee for several helpful suggestions.

\section{Preliminaries}

Closely related to minimal surfaces are harmonic maps. If we fix a conformal structure $\omega$ on a closed oriented surface $\Sigma$, we can define the $\omega$-energy

$$
E_{\omega}: \operatorname{Map}(\Sigma, M) \rightarrow \mathbb{R} \quad \text { by } \quad E_{\omega}(f)=\frac{1}{2} \int_{\Sigma}|d f|^{2} d A .
$$

Here the length of $d f$ and the area element $d A$ are calculated with respect to the given Riemannian metric on $M$ and any metric on $\Sigma$ within the conformal equivalence class defined by $\omega$. A critical point for $E_{\omega}$ is called an $\omega$-harmonic map. In terms of a complex parameter $z$, an $\omega$-harmonic map can be regarded as a solution to the equation,

$$
\frac{D}{\partial \bar{z}}\left(\frac{\partial f}{\partial z}\right)=0 ; \text { thus } \frac{\partial f}{\partial z}
$$

can be regarded as a holomorphic section of $\mathbf{E}=f^{*} T M \otimes \mathbb{C}$ when $\mathbf{E}$ is given the holomorphic structure defined by the Levi-Civita connection $D$; see Donaldson and Kronheimer [2], Proposition 2.1.56.

If $(f, \omega)$ is a crtical point for the two-variable energy (1), the map $f$ is not only $\omega$-harmonic, but also $\omega$-conformal, that is

$$
\left\langle\frac{\partial f}{\partial z}, \frac{\partial f}{\partial z}\right\rangle=0
$$

The only possible singularities of nonconstant conformal harmonic maps are branch points; each such branch point $p$ lies in an open neighborhood $U \subset \Sigma$ such that

$$
\frac{\partial f}{\partial z} \mid U=z^{\nu} g, \quad g \in \Gamma(\mathbf{E} \mid U), \quad g(p) \neq 0,
$$

where $z$ is a complex coordinate such that $z(p)=0$ and $\nu$ is a positive integer called the branching order at $p$. The locally defined sections $\partial f / \partial z$ and $g$ (near branch points) generate a line bundle $\mathbf{L} \subset \mathbf{E}$. The tangential component of $\mathbf{E}$ is

$$
\left(f^{*} T M \otimes \mathbb{C}\right)^{\top}=\mathbf{L} \oplus \overline{\mathbf{L}} .
$$

It follows from (3) that $\mathbf{L}$ is a holomorphic subbundle of $\mathbf{E}$. When $f$ has branch points, $\mathbf{L}$ is not the holomorphic tangent bundle to $\Sigma$ and in fact $c_{1}(\mathbf{L})([\Sigma])$ is $2-2 g$ plus the total branching order. 
A standard calculation shows that the second variation for $\omega$-energy is given by

$$
d^{2} E_{\omega}(f)(X, X)=\int_{\Sigma}\left[\|D X\|^{2}-\langle\mathcal{K}(X), X\rangle\right] d A,
$$

where in terms of the complex parameter $z=x_{1}+i x_{2}$ on $\Sigma$,

$$
\|D X\|^{2}=\frac{1}{\lambda^{2}}\left[\left\|\frac{D X}{\partial x_{1}}\right\|^{2}+\left\|\frac{D X}{\partial x_{2}}\right\|^{2}\right]
$$

and

$$
\langle\mathcal{K}(X), X\rangle=\frac{1}{\lambda^{2}}\left[\left\langle R\left(X, \frac{\partial f}{\partial x_{1}}\right) \frac{\partial f}{\partial x_{1}}, X\right\rangle+\left\langle R\left(X, \frac{\partial f}{\partial x_{2}}\right) \frac{\partial f}{\partial x_{2}}, X\right\rangle\right],
$$

$R$ being the Riemann-Christoffel curvature tensor of $M$. This second variation formula can be put in complex form as described in [7]:

$$
d^{2} E_{\omega}(f): \Gamma(\mathbf{E}) \times \Gamma(\mathbf{E}) \longrightarrow \mathbb{C}
$$

is given by the formula

$$
d^{2} E_{\omega}(f)(Z, \bar{Z})=4 \int_{\Sigma}\left[\left|\frac{D Z}{\partial \bar{z}}\right|^{2}-\left\langle R\left(Z, \frac{\partial f}{\partial z}\right) \frac{\partial f}{\partial \bar{z}}, \bar{Z}\right\rangle\right] d x d y
$$

when $z=x+i y$ is a complex parameter on $\Sigma$ and $R$ is the Riemann Christoffel curvature tensor of $M$. We can of course replace $z$ by $\bar{z}$ in this formula and obtain

$$
d^{2} E_{\omega}(f)(Z, \bar{Z})=4 \int_{\Sigma}\left[\left|\frac{D Z}{\partial z}\right|^{2}-\left\langle R\left(Z, \frac{\partial f}{\partial \bar{z}}\right) \frac{\partial f}{\partial z}, \bar{Z}\right\rangle\right] d x d y .
$$

Note that in both cases the integrands are independent of the choice of complex parameter on $\Sigma$ as well as the action of the diffeomorphism group on $\Sigma$.

\section{Second variation of energy}

In considering second variation of the two-variable energy (1), it is helpful to be explicit regarding dependence on the metric on $\Sigma$.

Let $\operatorname{Met}(\Sigma)$ denote the space of smooth Riemannian metrics on the compact oriented surface $\Sigma, \operatorname{Met}(M)$ the space of Riemannian metrics on an $n$ dimensional ambient manifold $M$. We can write a typical element $\eta \in \operatorname{Met}(\Sigma)$ in terms of fixed coordinates $\left(x_{1}, x_{2}\right)$ on $\Sigma$ as

$$
\eta=\sum_{a, b=1}^{2} \eta_{a b} d x_{a} d x_{b}, \quad \text { and let } \quad\left(\eta^{a b}\right)=\left(\eta_{a b}\right)^{-1} .
$$


We can then define the energy function

$$
E: \operatorname{Map}(\Sigma, M) \times \operatorname{Met}(\Sigma) \times \operatorname{Met}(M) \longrightarrow \mathbb{R}
$$

by means of the coordinate formula

$$
E(f, \eta, g)=\frac{1}{2} \int_{\Sigma} \sum_{a, b} \eta^{a b} \sqrt{\operatorname{det}\left(\eta_{a b}\right)}\left\langle\frac{\partial f}{\partial x_{a}}, \frac{\partial f}{\partial x_{b}}\right\rangle d x_{1} d x_{2}
$$

where

$$
\left\langle\frac{\partial f}{\partial x_{a}}, \frac{\partial f}{\partial x_{b}}\right\rangle=g\left(\frac{\partial f}{\partial x_{a}}, \frac{\partial f}{\partial x_{b}}\right),
$$

the integrand being independent of the choice of local coordinates.

We can fix the metric $g \in \operatorname{Met}(M)$, and consider the function

$$
E_{g}: \operatorname{Map}(\Sigma, M) \times \operatorname{Met}(\Sigma) \longrightarrow \mathbb{R}, \quad E_{g}(f, \eta)=E(f, \eta, g) .
$$

One can show that a critical point for the energy $(8)$ is a pair $(f, \eta)$ where $f$ is harmonic, as well as conformal with respect to the metric $\eta$ on $\Sigma$ and the fixed metric $\langle\cdot, \cdot\rangle$ on $M$. Moreover, one verifies directly that the energy $E$ does not change when the metric $\eta$ is multiplied by a conformal factor, that is $E$ is invariant under Weyl rescalings.

A direct calculation shows that the second derivative of energy at a critical point for the energy is a sum of three terms. If $X$ is a section of $f^{*} T M$ and $\dot{\eta}$ is a trace-free variation of the metric on $\Sigma$,

$$
\begin{aligned}
& d^{2} E_{g}(f, \eta)((X, \dot{\eta}),(X, \dot{\eta}))=d^{2} E_{\omega}(f)(X, X) \\
& \quad+\left.2 \int_{\Sigma} \sum_{a, b} \frac{d}{d t}\left(\eta^{a b} \sqrt{\operatorname{det}\left(\eta_{a b}\right)}\right)\right|_{t=0}\left\langle\frac{D X}{\partial x_{a}}, \frac{\partial f}{\partial x_{b}}\right\rangle d x_{1} d x_{2} \\
& \quad+\left.\frac{1}{2} \int_{\Sigma} \sum_{a, b} \frac{d^{2}}{d t^{2}}\left(\eta^{a b} \sqrt{\operatorname{det}\left(\eta_{a b}\right)}\right)\right|_{t=0}\left\langle\frac{\partial f}{\partial x_{a}}, \frac{\partial f}{\partial x_{b}}\right\rangle d x_{1} d x_{2}
\end{aligned}
$$

We can think of these three terms as second-order partial derivatives in the three directions:

$$
\operatorname{Map}(\Sigma, M) \times \operatorname{Map}(\Sigma, M), \quad \operatorname{Map}(\Sigma, M) \times \operatorname{Met}(\Sigma), \quad \operatorname{Met}(\Sigma) \times \operatorname{Met}(\Sigma) .
$$

As mentioned in the previous section, the first term in (9) is

$$
\int_{\Sigma}\left[\left\|\frac{D X}{\partial x_{1}}\right\|^{2}+\left\|\frac{D X}{\partial x_{2}}\right\|^{2}\right] d x_{1} d x_{2}-\int_{\Sigma}\langle\mathcal{K}(X), X\rangle d A .
$$

To evaluate the other two terms, we take a metric variation of the form

$$
\eta_{a b}(t)=\lambda^{2} \delta_{a b}+t \dot{\eta}_{a b}
$$


where we can assume that $\left(\dot{\eta}_{a b}\right)$ satisfies the trace-free condition $\dot{\eta}_{11}+\dot{\eta}_{22}=0$; the trace term $\dot{\eta}_{11}+\dot{\eta}_{22}$ does not affect the energy, and if we were to include it in the calculation its contribution would simply cancel out of the formula in the end. Differentiation then yields

$$
\left.\frac{d}{d t} \eta(t)\right|_{t=0}=0,\left.\quad \frac{d^{2}}{d t^{2}} \eta(t)\right|_{t=0}=2 \dot{\eta}_{11} \dot{\eta}_{22}-2 \dot{\eta}_{12} \dot{\eta}_{12}
$$

where we use the symbol $\eta$ to denote $\operatorname{det}\left(\eta_{a b}\right)$. Using the formulae

$$
\frac{d}{d t}\left(\eta^{-1 / 2}\right)=-\frac{1}{2} \eta^{-3 / 2} \frac{d \eta}{d t}, \quad \frac{d^{2}}{d t^{2}}\left(\eta^{-1 / 2}\right)=-\frac{1}{2} \eta^{-3 / 2} \frac{d^{2} \eta}{d t^{2}}+\frac{3}{4} \eta^{-5 / 2}\left(\frac{d \eta}{d t}\right)^{2}
$$

we can differentiate the components appearing in (7),

$$
\left(\begin{array}{cc}
\sqrt{\eta} \eta^{11} & \sqrt{\eta} \eta^{12} \\
\sqrt{\eta} \eta^{21} & \sqrt{\eta} \eta^{22}
\end{array}\right)=\eta^{-1 / 2}\left(\begin{array}{cc}
\eta_{22} & -\eta_{12} \\
-\eta_{21} & \eta_{11}
\end{array}\right)
$$

obtaining

$$
\frac{d}{d t}\left(\begin{array}{cc}
\sqrt{\eta} \eta^{11} & \sqrt{\eta} \eta^{12} \\
\sqrt{\eta} \eta^{21} & \sqrt{\eta} \eta^{22}
\end{array}\right)=\frac{d}{d t}\left(\eta^{-1 / 2}\right)\left(\begin{array}{cc}
\eta_{22} & -\eta_{12} \\
-\eta_{21} & \eta_{11}
\end{array}\right)-\eta^{-1 / 2}\left(\begin{array}{ll}
\dot{\eta}_{11} & \dot{\eta}_{12} \\
\dot{\eta}_{21} & \dot{\eta}_{22}
\end{array}\right),
$$

with a similar result for the second derivative. Evaluation at $t=0$ and use of (11) yields the results

$$
\begin{gathered}
\left.\frac{d}{d t}\left(\sqrt{\eta} \eta^{a b}\right)\right|_{t=0}=-\lambda^{-2} \dot{\eta}_{a b}, \\
\left.\frac{d^{2}}{d t^{2}}\left(\sqrt{\eta} \eta^{a b}\right)\right|_{t=0}=\lambda^{-4}\left(\dot{\eta}_{11}^{2}+\dot{\eta}_{12}^{2}\right) \delta_{a b} .
\end{gathered}
$$

It now follows from (12) that the second term in (9) is

$$
-2 \int_{\Sigma} \sum_{a, b} \frac{\dot{\eta}_{a b}}{\lambda^{2}}\left\langle\frac{D X}{\partial x_{a}}, \frac{\partial f}{\partial x_{b}}\right\rangle d x_{1} d x_{2}
$$

while from (13) and the trace-free condition we conclude that the third term is

$$
\int_{\Sigma} \frac{1}{\lambda^{4}}\left(\dot{\eta}_{11}^{2}+\dot{\eta}_{12}^{2}\right) \sigma^{2} d x_{1} d x_{2}=\frac{1}{2} \int_{\Sigma} \sum_{a, b}\left|\frac{\dot{\eta}_{a b}}{\lambda^{2}}\right|^{2}\left\|\frac{\partial f}{\partial x_{b}}\right\|^{2} d x_{1} d x_{2} .
$$

Adding the three terms together yields our second variation formula

$$
\begin{aligned}
& d^{2} E_{g}(f, \eta)((X, \dot{\eta}),(X, \dot{\eta}))=d^{2} E_{\omega, g}(f)(X, X) \\
&-2 \int_{\Sigma} \sum_{a, b} \frac{\dot{\eta}_{a b}}{\lambda^{2}}\left\langle\frac{D X}{\partial x_{a}}, \frac{\partial f}{\partial x_{b}}\right\rangle d x_{1} d x_{2} \\
& \quad+\int_{\Sigma} \frac{1}{\lambda^{4}}\left(\dot{\eta}_{11}^{2}+\dot{\eta}_{12}^{2}\right) \sigma^{2} d x_{1} d x_{2},
\end{aligned}
$$


where

$$
\sigma^{2}=\left|\frac{\partial f}{\partial x_{1}}\right|^{2}=\left|\frac{\partial f}{\partial x_{2}}\right|^{2}=2\left|\frac{\partial f}{\partial z}\right|^{2} .
$$

The group $\operatorname{Diff}^{+}(\Sigma)$ of orientation-preserving diffeomorphisms of $\Sigma$ acts on $\operatorname{Met}(\Sigma)$ by

$$
(\eta, \phi) \in \operatorname{Met}(\Sigma) \times \operatorname{Diff}^{+}(\Sigma) \quad \mapsto \quad \phi^{*} \eta \in \operatorname{Met}(\Sigma) .
$$

This group also acts on $\operatorname{Map}(\Sigma, M)$ by $(f, \phi) \mapsto f \circ \phi$, and the energy $E$ is invariant under the product action

$$
((f, \eta), \phi) \in \operatorname{Map}(\Sigma, M) \times \operatorname{Met}(\Sigma) \times \operatorname{Diff}^{+}(\Sigma) \quad \mapsto \quad\left(f \circ \phi, \phi^{*} \eta\right) .
$$

As mentioned before, the energy is also invariant under Weyl rescalings of the metric, $\left(\eta_{a b}\right) \rightarrow\left(\lambda^{2} \eta_{a b}\right)$, so the energy function (8) has an infinite-dimensional symmetry group and a corresponding infinite-dimensional family of Jacobi fields. To simplify the analysis, we let $\operatorname{Conf}(\Sigma)$ denote the space of equivalence classes of metrics under Weyl rescalings, and note that there is an isomorphism between $\operatorname{Conf}(\Sigma)$ and the space $\operatorname{Met}_{0}(\Sigma)$, the subspace of metrics on $\Sigma$ which have constant curvature and total area one. We can thus eliminate the Weyl rescalings by restricting $E$ to $\operatorname{Met}_{0}(\Sigma)$. To eliminate the symmetries coming from the diffeomorphism group, we can divide out by the normal subgroup $\operatorname{Diff}_{0}(\Sigma)$ of $\operatorname{Diff}^{+}(\Sigma)$ consisting of diffeomorphisms isotopic to the identity. The energy descends to a function defined on the quotient

$$
\frac{\operatorname{Map}(\Sigma, M) \times \operatorname{Conf}(\Sigma)}{\operatorname{Diff}_{0}(\Sigma)} \cong \operatorname{Map}(\Sigma, M) \times \mathcal{T},
$$

an isomorphism between the two sides being established by choice of a section of the action of $\operatorname{Conf}(\Sigma) \cong \operatorname{Diff}_{0}(\Sigma)$ on $\operatorname{Met}_{0}(\Sigma)$. (See Chapter 2 of [9] for construction of such a section.) The resulting map

$$
E_{g}: \operatorname{Map}(\Sigma, M) \times \mathcal{T} \longrightarrow \mathbb{R}
$$

has a finite-dimensional group of symmetries and is well-adapted to study by techniques from global analysis.

\section{Complexification}

To best understand the complex form of the second variation formula (16), it is convenient to describe the complexification of the tangent space to the space of metrics on $\Sigma$ in terms of canonical bundles. If $\operatorname{Met}(\Sigma)$ denotes the space of Riemannian metrics on a closed oriented surface $\Sigma$, and $\eta$ is an element of $\operatorname{Met}(\Sigma)$,

$$
T_{\eta}(\operatorname{Met}(\Sigma)) \otimes \mathbb{C}=\mathbf{K}^{(2,0)} \oplus \mathbf{K}^{(1,1)} \oplus \mathbf{K}^{(0,2)},
$$

where $\mathbf{K}$ is the canonical bundle over $\Sigma, \overline{\mathbf{K}}$ is its conjugate and

$$
\mathbf{K}^{(p, q)}=\mathbf{K}^{p} \otimes \overline{\mathbf{K}}^{q} .
$$


Of course, the holomorphic tangent bundle of $\Sigma$ is isomorphic to $\overline{\mathbf{K}}$. Covariant differentiations yield operators

$$
D^{\prime}: \Gamma\left(\mathbf{K}^{(p, q)}\right) \rightarrow \Gamma\left(\mathbf{K}^{(p+1, q)}\right), \quad D^{\prime \prime}: \Gamma\left(\mathbf{K}^{(p, q)}\right) \rightarrow \Gamma\left(\mathbf{K}^{(p, q+1)}\right) .
$$

Note that sections of the summand $\mathbf{K}^{(2,0)}$ are quadratic differentials on $\Sigma$.

The real inner product on $T \Sigma$ extends to a complex bilinear inner product $\langle\cdot, \cdot\rangle$ on the sum of all $\mathbf{K}^{(p, q)}$ 's, which pairs $\mathbf{K}^{(p, q)}$ with $\mathbf{K}^{(q, p)}$, or to a Hermitian inner product with respect to which all the $\mathbf{K}^{(p, q)}$ 's are orthogonal. The real structure determines a conjugation $C: \mathbf{K}^{(p, q)} \rightarrow \mathbf{K}^{(q, p)}$.

In the context of Fréchet manifolds, the Lie algebra of the group Diff $(\Sigma)$ of diffeomorphisms of $\Sigma$ is just the space of vector fields on $\Sigma$. Complex-valued vector fields are sections of $\mathbf{K} \oplus \overline{\mathbf{K}}$, and as described in [1], the action of $\Gamma(\mathbf{K} \oplus \overline{\mathbf{K}})$ on $T_{\eta}(\operatorname{Met}(\Sigma))$ is given by

$$
\begin{aligned}
& D^{\prime}+D^{\prime \prime}: \Gamma\left(\mathbf{K}^{(1,0)}\right) \rightarrow \Gamma\left(\mathbf{K}^{(2,0)}\right) \oplus \Gamma\left(\mathbf{K}^{(1,1)}\right), \\
& D^{\prime}+D^{\prime \prime}: \Gamma\left(\mathbf{K}^{(0,1)}\right) \rightarrow \Gamma\left(\mathbf{K}^{(1,1)}\right) \oplus \Gamma\left(\mathbf{K}^{(0,2)}\right) .
\end{aligned}
$$

Thus for example, if one restricts attention to the holomorphic tangent bundle $\overline{\mathbf{K}}$, the key piece of this action is

$$
D^{\prime \prime}: Z \mapsto \frac{D Z}{\partial \bar{z}} d \bar{z},
$$

The other component $D^{\prime}(Z)$ simply affects the scale factor in the metric represented by $\mathbf{K}^{(1,1)}$. The cokernel of $D^{\prime}: \Gamma\left(\mathbf{K}^{(1,0)}\right) \rightarrow \Gamma\left(\mathbf{K}^{(2,0)}\right)$ can be thought of as the cotangent space to Teichmüller space consisting of holomorphic quadratic differentials, which is isomorphic to the tangent space consisting of extremal Beltrami differentials.

To analyze the second term in the variation formula (16), we set $\phi=$ $(1 / 2)\left(\dot{\eta}_{11}-\dot{\eta}_{22}\right)-i \dot{\eta}_{12}$, and take the complex bilinear inner product between the two expressions

$$
\sum \frac{D X}{\partial x_{a}} d x_{a}=\frac{D X}{\partial z} d z+\frac{D X}{\partial \bar{z}} d \bar{z}
$$

and

$$
\sum \dot{\eta}_{a b} \frac{\partial f}{\partial x_{b}} d x_{a}=\phi \frac{\partial f}{\partial \bar{z}} d z+\bar{\phi} \frac{\partial f}{\partial z} d \bar{z} .
$$

Using the fact that $\left\langle d x_{a}, d x_{b}\right\rangle=\left(1 / \lambda^{2}\right) \delta_{a b}$, we see that the second term of (16) is -2 times the inner product of the left hand sides of (18) and (19). Thus, taking the inner product of the right-hand sides, we see that the second term is

$$
-4 \int_{\Sigma}\left\langle\frac{D X}{\partial z}, \frac{\bar{\phi}}{\lambda^{2}} \frac{\partial f}{\partial z}\right\rangle d x_{1} d x_{2}-4 \int_{\Sigma}\left\langle\frac{D X}{\partial \bar{z}}, \frac{\phi}{\lambda^{2}} \frac{\partial f}{\partial \bar{z}}\right\rangle d x_{1} d x_{2} .
$$

Here $\phi$ transforms under change of complex parameter in such a way that

$$
\phi d z^{2} \leftrightarrow \frac{2 \bar{\phi}}{\lambda^{2}} \frac{d \bar{z}}{d z}
$$


is a quadratic or Beltrami differential, a section of $\mathbf{K}^{(2,0)}$ or $\mathbf{K}^{(-1,1)}$ respectively. The third term in (16) is

$$
\int_{\Sigma} \frac{1}{\lambda^{4}}\left(\left|\dot{\eta}_{11}\right|^{2}+\left|\dot{\eta}_{12}\right|^{2}\right) \sigma^{2} d x_{1} d x_{2}=\int_{\Sigma}\left(\left|\frac{\bar{\phi}}{\lambda^{2}} \frac{\partial f}{\partial z}\right|^{2}+\left|\frac{\phi}{\lambda^{2}} \frac{\partial f}{\partial \bar{z}}\right|^{2}\right) d x_{1} d x_{2} .
$$

Thus if $Z$ is a section of $f^{*} T M \otimes \mathbb{C}$,

$$
\begin{array}{r}
d^{2} E(f, \omega)((Z, \dot{\eta}),(\bar{Z}, \dot{\eta}))=d^{2} E_{\omega}(f)(Z, \bar{Z}) \\
-4 \operatorname{Re} \int_{\Sigma}\left\langle\frac{D Z}{\partial \bar{z}}, \frac{\phi}{\lambda^{2}} \frac{\partial f}{\partial \bar{z}}\right\rangle d x_{1} d x_{2}-4 \operatorname{Re} \int_{\Sigma}\left\langle\frac{D Z}{\partial z}, \frac{\bar{\phi}}{\lambda^{2}} \frac{\partial f}{\partial z}\right\rangle d x_{1} d x_{2} \\
\quad+\int_{\Sigma}\left(\left|\frac{\bar{\phi}}{\lambda^{2}} \frac{\partial f}{\partial z}\right|^{2}+\left|\frac{\phi}{\lambda^{2}} \frac{\partial f}{\partial \bar{z}}\right|^{2}\right) d x_{1} d x_{2}
\end{array}
$$

To allow $\dot{\eta}_{a b}$ to be complex, we replace $\phi$ by $\psi$ in the first term on the right in (19), which corresponds to specifying two quadratic differentials $\phi d z^{2}$ and $\bar{\psi} d \bar{z}^{2}$, sections of $\mathbf{K}^{(2,0)}$ and $\mathbf{K}^{(0,2)}$ respectively. Note that

$$
\left(Z, \phi d z^{2}, \bar{\psi} d \bar{z}^{2}\right) \in\left(T_{f} \operatorname{Map}(\Sigma, M) \otimes \mathbb{C}\right) \oplus \Gamma\left(\mathbf{K}^{(2,0)}\right) \oplus \Gamma\left(\mathbf{K}^{(0,2)}\right),
$$

has conjugate $\left(\bar{Z}, \psi d z^{2}, \bar{\phi} d \bar{z}^{2}\right)$, since conjugation interchanges $\mathbf{K}^{(2,0)}$ and $\mathbf{K}^{(0,2)}$. The second variation formula now becomes

$$
\begin{array}{r}
d^{2} E(f, \omega)\left(\left(Z, \phi d z^{2}, \bar{\psi} d \bar{z}^{2}\right),\left(\bar{Z}, \psi d z^{2}, \bar{\phi} d \bar{z}^{2}\right)\right)=d^{2} E_{\omega}(f)(Z, \bar{Z}) \\
-4 \operatorname{Re} \int_{\Sigma}\left\langle\frac{D Z}{\partial \bar{z}}, \frac{\phi}{\lambda^{2}} \frac{\partial f}{\partial \bar{z}}\right\rangle d x_{1} d x_{2}-4 \operatorname{Re} \int_{\Sigma}\left\langle\frac{D Z}{\partial z}, \frac{\bar{\psi}}{\lambda^{2}} \frac{\partial f}{\partial z}\right\rangle d x_{1} d x_{2} \\
+\int_{\Sigma}\left(\left|\frac{\bar{\phi}}{\lambda^{2}} \frac{\partial f}{\partial z}\right|^{2}+\left|\frac{\psi}{\lambda^{2}} \frac{\partial f}{\partial \bar{z}}\right|^{2}\right) d x_{1} d x_{2} .
\end{array}
$$

Substitution of (5) into this formula yields:

Second Variation Theorem. The second variation of the two-variable energy $E$ is given by

$$
\begin{aligned}
d^{2} E(f, \omega)( & \left.\left(Z, \phi d z^{2}, \bar{\psi} d \bar{z}^{2}\right),\left(\bar{Z}, \psi d z^{2}, \bar{\phi} d \bar{z}^{2}\right)\right) \\
=\int_{\Sigma} & {\left[\left|2 \frac{D Z}{\partial \bar{z}}-\frac{\bar{\phi}}{\lambda^{2}} \frac{\partial f}{\partial z}\right|^{2}-4\left\langle R\left(Z, \frac{\partial f}{\partial z}\right) \frac{\partial f}{\partial \bar{z}}, \bar{Z}\right\rangle\right] d x_{1} d x_{2} } \\
& \quad-4 \operatorname{Re} \int_{\Sigma}\left\langle\frac{D Z}{\partial z}, \frac{\bar{\psi}}{\lambda^{2}} \frac{\partial f}{\partial z}\right\rangle d x_{1} d x_{2}+\int_{\Sigma}\left|\frac{\psi}{\lambda^{2}} \frac{\partial f}{\partial \bar{z}}\right|^{2} d x_{1} d x_{2} .
\end{aligned}
$$

Here $Z$ is a section of $f^{*} T M \otimes \mathbb{C}$ and $\left(\phi d z^{2}, \bar{\psi} d \bar{z}^{2}\right)$ is an element of $T_{\omega} \mathcal{T} \otimes \mathbb{C}$. 
Had we used (6) instead, we would have obtained

$$
\begin{aligned}
d^{2} E(f, \omega)( & \left.\left(Z, \phi d z^{2}, \bar{\psi} d \bar{z}^{2}\right),\left(\bar{Z}, \psi d z^{2}, \bar{\phi} d \bar{z}^{2}\right)\right) \\
=\int_{\Sigma} & {\left[\left|2 \frac{D Z}{\partial z}-\frac{\psi}{\lambda^{2}} \frac{\partial f}{\partial \bar{z}}\right|^{2}-4\left\langle R\left(Z, \frac{\partial f}{\partial \bar{z}}\right) \frac{\partial f}{\partial z}, \bar{Z}\right\rangle\right] d x_{1} d x_{2} } \\
& \quad-4 \operatorname{Re} \int_{\Sigma}\left\langle\frac{D Z}{\partial \bar{z}}, \frac{\phi}{\lambda^{2}} \frac{\partial f}{\partial \bar{z}}\right\rangle d x_{1} d x_{2}+\int_{\Sigma}\left|\frac{\bar{\phi}}{\lambda^{2}} \frac{\partial f}{\partial z}\right|^{2} d x_{1} d x_{2} .
\end{aligned}
$$

From these formulae, we see quite clearly how the action of the diffeomorphism group enters into the second variation formula, complexifications of infinitesimal diffeomorphisms being sections of $\mathbf{L} \oplus \overline{\mathbf{L}}$ or of $\mathbf{K}^{(0,1)} \oplus \mathbf{K}^{(1,0)}$.

\section{Relationship to second variation of area}

We now describe the relationship between (16) and (24) and the classical formulae presented in Simons [10] and Micallef and Wolfson [8] for second variation of area under normal variations. Of course, if $f: \Sigma \rightarrow M$ is an immersion, second derivative of area along normal deformations must agree with the second derivative of energy along deformations through an appropriate family of conformal harmonic maps, in which the conformal structure changes in accordance with the immersion.

To see how this works out, we suppose that $X^{\perp}$ is a section of the normal bundle $\left(f^{*} T M\right)^{\perp}$ to $f$, and let

$$
\sum_{a, b=1}^{2} \eta_{a b} d x_{a} d x_{b}=\lambda^{2}\left(d x_{1}^{2}+d x_{2}^{2}\right)
$$

denote the metric on $\Sigma$ induced by $f$, described in terms of isothermal coordinates $\left(x_{1}, x_{2}\right)$ on $\Sigma$. A variation in the direction of $X^{\perp}$ will produce a corresponding variation $\sigma\left(X^{\perp}\right)=\left(\dot{\eta}_{a b}\right)$ in the induced metric on $\Sigma$, which is given by the formula

$$
\dot{\eta}_{a b}=\left[\left\langle\frac{D X^{\perp}}{\partial x_{a}}, \frac{\partial f}{\partial x_{b}}\right\rangle+\left\langle\frac{\partial f}{\partial x_{a}}, \frac{D X^{\perp}}{\partial x_{b}}\right\rangle\right]=-2\left\langle X^{\perp}, \frac{D}{\partial x_{a}}\left(\frac{\partial f}{\partial x_{b}}\right)\right\rangle,
$$

the last equality using the fact that $X^{\perp}$ is perpendicular to $\partial f / \partial x_{1}$ and $\partial f / \partial x_{2}$. Note that since $f$ is harmonic, it follows from the right-hand expression that $\dot{\eta}_{11}+\dot{\eta}_{22}=0$, so the variation is infinitesimally area preserving. Since the deformation $\left(X^{\perp}, \sigma\left(X^{\perp}\right)\right)$ is through conformal maps, it is to be expected that this will result in the most negative possible second variation.

It follows from (26) that

$$
\left\langle\frac{D X^{\perp}}{\partial x_{a}}, \frac{\partial f}{\partial x_{b}}\right\rangle=\frac{1}{2} \dot{\eta}_{a b}
$$


and hence the second and third terms (14) and (15) in the second variation formula are

$$
-\int_{\Sigma} \sum_{a, b}\left|\frac{\dot{\eta}_{a b}}{\lambda}\right|^{2} d x_{1} d x_{2} \quad \text { and } \quad \frac{1}{2} \int_{\Sigma} \sum_{a, b}\left|\frac{\dot{\eta}_{a b}}{\lambda}\right|^{2} d x_{1} d x_{2}
$$

respectively. It follows that the second variation is given by

$$
\begin{aligned}
d^{2} E(f, \eta)\left(\left(X^{\perp}, \dot{\eta}\right),\left(X^{\perp}, \dot{\eta}\right)\right)= & \int_{\Sigma} \sum_{a}\left\|D X^{\perp}\right\|^{2} d A \\
& -\frac{1}{2} \int_{\Sigma} \sum_{a, b}\left|\frac{\dot{\eta}_{a b}}{\lambda^{2}}\right|^{2} d A-\int_{\Sigma}[\langle\mathcal{K}(X), X\rangle] d A .
\end{aligned}
$$

A direct calculation shows that

$$
\sum_{a, b}\left|\frac{\dot{\eta}_{a b}}{\lambda^{2}}\right|^{2}=4 \sum_{a, b}\left|\frac{1}{\lambda^{2}}\left\langle\frac{D X^{\perp}}{\partial x_{a}}, \frac{\partial f}{\partial x_{b}}\right\rangle\right|^{2}=4\left\|\left(D X^{\perp}\right)^{\top}\right\|^{2},
$$

from which we obtain the real version of the classical formula for second variation of area ([10], Theorem 3.2.2 or [4], Theorem 32):

$$
\begin{aligned}
& d^{2} E(f, \omega)\left(\left(X^{\perp}, \sigma\left(X^{\perp}\right)\right),\left(X^{\perp}, \sigma\left(X^{\perp}\right)\right)\right) \\
&=\int_{\Sigma}\left[\left\|D X^{\perp}\right\|^{2}-2\left\|\left(D X^{\perp}\right)^{\top}\right\|^{2}-\left\langle\mathcal{K}\left(X^{\perp}\right), X^{\perp}\right\rangle\right] d A \\
&=\int_{\Sigma}\left[\left\|\left(D X^{\perp}\right)^{\perp}\right\|^{2}-\left\langle\mathcal{B}\left(X^{\perp}\right)+\mathcal{K}\left(X^{\perp}\right), X^{\perp}\right\rangle\right] d A,
\end{aligned}
$$

where

$$
\left\langle\mathcal{B}\left(X^{\perp}\right), X^{\perp}\right\rangle=\left\|\left(D X^{\perp}\right)^{\top}\right\|^{2},
$$

a term which, as one easily verifies, depends only on the second fundamental form of $f$. From this second variation formula, we obtain a formally self-adjoint Jacobi operator

$$
L^{\perp}: \Gamma\left(\left(f^{*} T M\right)^{\perp}\right) \rightarrow \Gamma\left(\left(f^{*} T M\right)^{\perp}\right),
$$

such that

$$
d^{2} E(f, \omega)\left(\left(X_{1}^{\perp}, \sigma\left(X_{1}^{\perp}\right)\right),\left(X_{2}^{\perp}, \sigma\left(X_{2}^{\perp}\right)\right)\right)=\int_{\Sigma}\left\langle L^{\perp}\left(X_{1}^{\perp}\right), X_{2}^{\perp}\right\rangle d A,
$$

which we call the normal Jacobi operator. We call a solution to $L^{\perp}\left(X^{\perp}\right)=0$ a normal Jacobi field.

How can we describe normal variations in the context of the complex vector bundle $\mathbf{E}$ ? The first step is to introduce a holomorphic bundle of codimension one in $\mathbf{E}$,

$$
\mathbf{L}^{\perp}=\{v \in \mathbf{E}:\langle v, w\rangle=0 \text { for all } w \in \mathbf{L}\} .
$$


We can then define the holomorphic normal bundle $\mathbf{N}(f)$ to $f$ by

$$
\mathbf{N}(f)=\mathbf{L}^{\perp} / \mathbf{L} .
$$

We can choose the first quadratic differential $\phi d z^{2}$ to cancel the tangential projection of $D Z^{\perp} / \partial \bar{z}$ and (24) becomes

$$
\begin{aligned}
d^{2} E(f, \omega)\left(\left(Z^{\perp}, \phi d z^{2}, \bar{\psi} d \bar{z}^{2}\right),\left(\bar{Z}^{\perp}, \psi d z^{2}, \bar{\phi} d \bar{z}^{2}\right)\right) & \\
=4 \int_{\Sigma} & {\left[\left|\left(\frac{D Z^{\perp}}{\partial \bar{z}}\right)^{\perp}\right|^{2}-\left\langle R\left(Z^{\perp}, \frac{\partial f}{\partial z}\right) \frac{\partial f}{\partial \bar{z}}, \bar{Z}^{\perp}\right\rangle\right] d x_{1} d x_{2} } \\
& -4 \operatorname{Re} \int_{\Sigma}\left\langle\frac{D Z^{\perp}}{\partial z}, \frac{\bar{\psi}}{\lambda^{2}} \frac{\partial f}{\partial z}\right\rangle d x_{1} d x_{2}+\int_{\Sigma}\left|\frac{\psi}{\lambda^{2}} \frac{\partial f}{\partial \bar{z}}\right|^{2} d x_{1} d x_{2} .
\end{aligned}
$$

Here $Z^{\perp}$ denotes a section of $\mathbf{N}(f)$ and diffeomorphism invariance ensures that we can assume that $\psi d z^{2}$ is a holomorphic differential.

The penultimate term in (29) can be interpreted in terms of the quadratic differential

$$
\zeta d z^{2}=-4\left\langle Z^{\perp}, \frac{D}{\partial z}\left(\frac{\partial f}{\partial z}\right)\right\rangle d z^{2}=4\left\langle\frac{D Z^{\perp}}{\partial z}, \frac{\partial f}{\partial z}\right\rangle d z^{2} .
$$

Then (29) becomes

$$
\begin{aligned}
& d^{2} E(f, \omega)\left(\left(Z^{\perp}, \phi d z^{2}, \bar{\psi} d \bar{z}^{2}\right),\left(\bar{Z}^{\perp}, \psi d z^{2}, \bar{\phi} d \bar{z}^{2}\right)\right) \\
&=4 \int_{\Sigma}\left[\left|\left(\frac{D Z^{\perp}}{\partial \bar{z}}\right)^{\perp}\right|^{2}-\left\langle R\left(Z^{\perp}, \frac{\partial f}{\partial z}\right) \frac{\partial f}{\partial \bar{z}}, \bar{Z}^{\perp}\right\rangle\right] d x_{1} d x_{2} \\
&-\operatorname{Re} \frac{\zeta \bar{\psi}}{\lambda^{4}} d A+\int_{\Sigma} \frac{|\psi|^{2}}{2 \lambda^{4}} d A,
\end{aligned}
$$

where we have assumed that the metric $\lambda^{2}\left(d x^{2}+d y^{2}\right)$ on $\Sigma$ is the metric $\sigma^{2}\left(d x^{2}+\right.$ $\left.d y^{2}\right)$ induced by $f$. The sum of the last two terms is minimized when $\zeta=\psi$, and then (30) becomes

$$
\begin{aligned}
& d^{2} E(f, \omega)\left(\left(Z^{\perp}, \phi d z^{2}, \bar{\psi} d \bar{z}^{2}\right),\left(\bar{Z}^{\perp}, \psi d z^{2}, \bar{\phi} d \bar{z}^{2}\right)\right) \\
& =4 \int_{\Sigma}\left[\left|\left(\frac{D Z^{\perp}}{\partial \bar{z}}\right)^{\perp}\right|^{2}-\left\langle R\left(Z^{\perp}, \frac{\partial f}{\partial z}\right) \frac{\partial f}{\partial \bar{z}}, \bar{Z}^{\perp}\right\rangle\right] d x_{1} d x_{2}-\frac{1}{2} \int_{\Sigma} \frac{|\zeta|^{2}}{\lambda^{4}} d A .
\end{aligned}
$$

Note that

$$
\left\langle\frac{\partial Z^{\perp}}{\partial z}, \frac{\partial f}{\partial \bar{z}}\right\rangle=-\left\langle Z^{\perp}, \frac{\partial^{2} f}{\partial z \partial \bar{z}}\right\rangle=0
$$


SO

$$
\left(\frac{\partial Z^{\perp}}{\partial z}\right)^{\top}=\frac{2}{\lambda^{2}}\left\langle\left(\frac{\partial Z^{\perp}}{\partial z}\right)^{\top}, \frac{\partial f}{\partial z}\right\rangle \frac{\partial f}{\partial \bar{z}}=-\frac{2}{\lambda^{2}}\left\langle Z^{\perp}, \frac{D}{\partial z}\left(\frac{\partial f}{\partial z}\right)\right\rangle \frac{\partial f}{\partial \bar{z}}
$$

and

$$
4\left|\left(\frac{\partial Z^{\perp}}{\partial z}\right)^{\top}\right|^{2} d x_{1} d x_{2}=4\left|-\frac{\zeta}{2 \lambda^{2}} \frac{\partial f}{\partial z}\right|^{2} d x_{1} d x_{2}=\frac{|\zeta|^{2}}{2 \lambda^{4}} d A
$$

so (30) can be rewritten as

$$
\begin{aligned}
& d^{2} E(f, \omega)\left(\left(Z^{\perp}, \phi d z^{2}, \bar{\psi} d \bar{z}^{2}\right),\left(\bar{Z}^{\perp}, \psi d z^{2}, \bar{\phi} d \bar{z}^{2}\right)\right) \\
& =4 \int_{\Sigma}\left[\left|\left(\frac{D Z^{\perp}}{\partial \bar{z}}\right)^{\perp}\right|^{2}-\left\langle R\left(Z^{\perp}, \frac{\partial f}{\partial z}\right) \frac{\partial f}{\partial \bar{z}}, \bar{Z}^{\perp}\right\rangle-\left|\left(\frac{\partial Z^{\perp}}{\partial z}\right)^{\top}\right|^{2}\right] d x_{1} d x_{2},
\end{aligned}
$$

which is just the formula for second variation of area presented by Micallef and Wolfson [8].

Invariance under diffeomorphisms of $\Sigma$ shows that all that really matters in (32) is the projection of $\zeta$ into the space of holomorphic quadratic differentials.

\section{Kähler metrics}

Variations with nontrivial tangential components make an important appearance in the theory of holomorphic curves in a Kähler manifold, or more generally, in an almost Kähler manifold (as studied in [5]). Recall that a Riemannian manifold $(M,\langle\cdot, \cdot\rangle)$ is called almost Hermitian if it is endowed with an almost complex structure $J$ such that

$$
\langle J v, J w\rangle=\langle v, w\rangle, \quad \text { for all } v, w \in T_{p} M .
$$

Given any almost Hermitian manifold $(M,\langle\cdot, \cdot\rangle, J)$ we define its Kähler form to be the two-form $\omega$ such that

$$
\omega(v, w)=\langle J v, w\rangle, \quad \text { for all } v, w \in T_{p} M .
$$

The almost Hermitian manifold $(M,\langle\cdot, \cdot\rangle, J)$ is said to be almost Kähler if this form is closed. It is said to be Kähler if the Kähler form is closed and $J$ is integrable, or equivalently, if $J$ is parallel with respect to the Levi-Civita connection $\nabla$.

If $(M,\langle\cdot, \cdot\rangle, J)$ is any Hermitian manifold, we can extend $\langle\cdot, \cdot\rangle$ and $J$ complex linearly to $T M \otimes \mathbb{C}$ and let

$$
\begin{aligned}
& T^{1,0} M=\{v \in T M \otimes \mathbb{C}: J v=\sqrt{-1} v\}, \\
& T^{0,1} M=\{v \in T M \otimes \mathbb{C}: J v=-\sqrt{-1} v\},
\end{aligned}
$$


called the holomorphic and antiholomorphic tangent bundles respectively. These bundles are interchanged by the conjugation operation $z \mapsto \bar{z}$ and are isotropicthat is

$$
v, w \in T_{p}^{1,0} M \quad \Rightarrow \quad\langle v, w\rangle=0 .
$$

Note that $(1 / 2)(\mathrm{id}-\sqrt{-1} J)$ and $(1 / 2)(\mathrm{id}+\sqrt{-1} J)$ are orthogonal projections taking sections of $T M \otimes \mathbb{C}$ into sections of $T^{1,0} M$ and $T^{0,1} M$, and hence if $f: \Sigma \rightarrow M$ is a smooth map,

$$
\left|\frac{\partial f}{\partial z}\right|^{2}=\left|\left(\frac{\partial f}{\partial z}\right)^{(1,0)}\right|^{2}+\left|\left(\frac{\partial f}{\partial z}\right)^{(0,1)}\right|^{2}
$$

where

$$
\left(\frac{\partial f}{\partial z}\right)^{(1,0)}=\frac{1}{2}\left(\frac{\partial f}{\partial z}-\sqrt{-1} J \frac{\partial f}{\partial z}\right), \quad\left(\frac{\partial f}{\partial z}\right)^{(0,1)}=\frac{1}{2}\left(\frac{\partial f}{\partial z}+\sqrt{-1} J \frac{\partial f}{\partial z}\right) .
$$

We say that $f: \Sigma \rightarrow M$ is $J$-holomorphic if

$$
J\left(\frac{\partial f}{\partial z}\right)=\sqrt{-1} \frac{\partial f}{\partial z} \quad \text { or equivalently, } \quad \frac{\partial f}{\partial z}=\left(\frac{\partial f}{\partial z}\right)^{(1,0)} .
$$

Such a map is holomorphic in the usual sense if $M$ is a Kähler manifold, and agrees in with the notion of $J$-holomorphic curves studied in symplectic geometry. Similarly, $f$ is anti-J-holomorphic if

$$
J\left(\frac{\partial f}{\partial z}\right)=-\sqrt{-1} \frac{\partial f}{\partial z} .
$$

In an almost Hermitian manifold, we can divide the energy,

$$
E(f, \omega)=2 \int_{\Sigma}\left|\frac{\partial f}{\partial z}\right|^{2} d x d y
$$

into two components, $E(f, \omega)=E^{\prime}(f, \omega)+E^{\prime \prime}(f, \omega)$, both nonnegative, where

$$
E^{\prime}(f, \omega)=2 \int_{\Sigma}\left|\left(\frac{\partial f}{\partial z}\right)^{(1,0)}\right|^{2} d x d y, \quad E^{\prime \prime}(f, \omega)=2 \int_{\Sigma}\left|\left(\frac{\partial f}{\partial z}\right)^{(0,1)}\right|^{2} d x d y
$$

Thus $(f, \omega)$ is $J$-holomorphic if and only if $E^{\prime \prime}(f, \omega)=0$. then

If $f: \Sigma \rightarrow M$ is a smooth map into an almost Hermitian manifold $(M,\langle\cdot, \cdot\rangle, J)$,

$$
E^{\prime}(f, \omega)=\frac{1}{2}\left(E(f, \omega)+\int_{\Sigma} f^{*} \omega\right), \quad E^{\prime \prime}(f, \omega)=\frac{1}{2}\left(E(f, \omega)-\int_{\Sigma} f^{*} \omega\right),
$$


where $\omega$ is the Kähler form. This fact is a direct consequence of the calculation:

$$
\begin{aligned}
2\left|\left(\frac{\partial f}{\partial z}\right)^{(1,0)}\right|^{2}=\left\langle\frac{\partial f}{\partial z}, \frac{\partial f}{\partial \bar{z}}\right\rangle-\frac{1}{2} & \left\langle\sqrt{-1} J \frac{\partial f}{\partial z}, \frac{\partial f}{\partial \bar{z}}\right\rangle \\
& =\frac{1}{4}\left[\left|\frac{\partial f}{\partial x}\right|^{2}+\left|\frac{\partial f}{\partial y}\right|^{2}\right]+\frac{1}{2} \omega\left(\frac{\partial f}{\partial x}, \frac{\partial f}{\partial y}\right) .
\end{aligned}
$$

If $(M,\langle\cdot, \cdot\rangle, J)$ is almost Kähler, the integral of $\omega$ is constant, and $E^{\prime}$ and $E^{\prime \prime}$ have exactly the same critical points as $E$; that is, their critical points are conformal harmonic maps. In this case, a $J$-holomorphic curve $(f, \omega)$ is both harmonic and conformal, the latter because $T^{1,0} M$ is isotropic. Moreover, a $J$-holomorphic curve is automatically stable and minimizes energy within its homotopy class.

If $M$ is Kähler, the holomorphic tangent and cotangent bundles are preserved by parallel translation, and hence if $Z$ and $W$ are sections of $T^{1,0} M$, then

$$
\left\langle\nabla_{X} \nabla_{Y} W, Z\right\rangle=0 \quad \text { for all } X \text { and } Y \quad \Rightarrow \quad\langle R(X, Y) W, Z\rangle=0 .
$$

Thus if we define the curvature operator

$$
\mathcal{R}: \Lambda^{2} T M \otimes \mathbb{C} \rightarrow \Lambda^{2} T M \otimes \mathbb{C} \quad \text { by } \quad\langle\mathcal{R}(X \wedge Y), Z \wedge W\rangle=\langle R(X, Y) W, Z\rangle,
$$

we see that $\mathcal{R}$ vanishes on $\Lambda^{2}\left(T^{1,0} M\right)$ and $\Lambda^{2}\left(T^{0,1} M\right)$.

Kähler metrics are far from being generic, and one expects nontrivial Jacobi fields along algebraic curves which admit nontrivial deformations; these are common in smooth algebraic varieties. Suppose that $f: \Sigma \rightarrow M$ is a holomorphic curve and $\left(Z, \phi d z^{2}\right)$ is a pair, where $Z$ is a section of the holomorphic tangent bundle $f^{*} T^{1,0} M$ and $\phi d z^{2}$ is a holomorphic quadratic differential which satisfy the equation

$$
\frac{D Z}{\partial \bar{z}}=\frac{\bar{\phi}}{2 \lambda^{2}} \frac{\partial f}{\partial z} .
$$

We can then set $\psi=0$ and obtain a Jacobi field $\left(Z, \phi d z^{2}, 0\right)$ for $(24)$. To see this, we need to check that

$$
d^{2} E(f, \omega)\left(\left(Z, \phi d z^{2}, 0\right),\left(\bar{Z}_{1}, \psi_{1} d z^{2}, \bar{\phi}_{1} d \bar{z}^{2}\right)\right)=0
$$

for all $\left(\bar{Z}_{1}, \psi_{1} d z^{2}, \bar{\phi}_{1} d \bar{z}^{2}\right)$. But the curvature term vanishes by the argument in the preceding paragraph. Thus the equation (24) implies that the only terms that could be nonzero are those which appear in

$$
d^{2} E(f, \omega)\left(\left(Z, \phi d z^{2}, 0\right),\left(0, \psi_{1} d z^{2}, 0\right)\right),
$$

and it is apparent from (33) that the only term in this expression that does not immediately vanish is

$$
-4 \operatorname{Re} \int_{\Sigma}\left\langle\frac{D Z}{\partial z}, \frac{\bar{\psi}_{1}}{\lambda^{2}} \frac{\partial f}{\partial z}\right\rangle d x_{1} d x_{2}
$$


This term vanishes because $f^{*} T^{1,0} M \oplus f^{*} T^{0,1} M$ is a parallel unitary direct sum decomposition of $\left(f^{*} T M\right) \otimes \mathbb{C}$, and $f^{*} T^{1,0} M$ is an isotropic subbundle of E which contains $\partial f / \partial z$.

In a deformation through algebraic curves, the quadratic differential in the Jacobi equation allows for a change in conformal structure on $\Sigma$.

\section{Tangential Jacobi fields}

The second variation formula for the two-variable energy gives rise to the possibility of tangential Jacobi fields, Jacobi fields which are tangential to the surface $\Sigma$. Suppose that $\left(Z, \phi d z^{2}\right)$ is a pair, where $Z$ is a section of the line bundle $\mathbf{L}$ described in $\S 2$ and $\phi d z^{2}$ is a holomorphic quadratic differential which satisfy the equation

$$
\frac{D Z}{\partial \bar{z}}=\frac{\bar{\phi}}{2 \lambda^{2}} \frac{\partial f}{\partial z} .
$$

We can then set $\psi=0$ and obtain a Jacobi field $\left(Z, \phi d z^{2}, 0\right)$ for $(24)$, by an argument almost identical to that presented in the previous section. Indeed, we need to check that

$$
d^{2} E(f, \omega)\left(\left(Z, \phi d z^{2}, 0\right),\left(\bar{Z}_{1}, \psi_{1} d z^{2}, \bar{\phi}_{1} d \bar{z}^{2}\right)\right)=0
$$

for all $\left(\bar{Z}_{1}, \psi_{1} d z^{2}, \bar{\phi}_{1} d \bar{z}^{2}\right)$. But the curvature term vanishes when $Z$ is a section of $\mathbf{L}$, no matter what $Z_{1}$ is, and (34) implies that the only terms that could be nonzero are those which appear in

$$
d^{2} E(f, \omega)\left(\left(Z, \phi d z^{2}, 0\right),\left(0, \psi_{1} d z^{2}, 0\right)\right) .
$$

Indeed, we see that the only term in this expression that does not immediately vanish is

$$
-4 \operatorname{Re} \int_{\Sigma}\left\langle\frac{D Z}{\partial z}, \frac{\bar{\psi}_{1}}{\lambda^{2}} \frac{\partial f}{\partial z}\right\rangle d x_{1} d x_{2}
$$

and this term vanishes because $\mathbf{L} \oplus \overline{\mathbf{L}}$ is a parallel decomposition of $\left(f^{*} T M\right)^{\top} \otimes \mathbb{C}$, the tangential part of $\left(f^{*} T M\right) \otimes \mathbb{C}$, and

$$
\left\langle\frac{\partial f}{\partial z}, \frac{\partial f}{\partial z}\right\rangle=0 \quad \text { or equivalently, }\langle\mathbf{L}, \mathbf{L}\rangle=0 .
$$

Conversely, it is not difficult to show that all Jacobi fields $\left(Z, \phi d z^{2}, \bar{\psi} d \bar{z}^{2}\right)$ in which $Z$ is a section of $\mathbf{L}$ are of the form $\left(Z, \phi d z^{2}, 0\right)$ where $\phi d z^{2}$ is a holomorphic quadratic differential which satisfies (34), or equivalently,

$$
D^{\prime \prime}(Z)=\iota\left(\bar{\phi} d \bar{z}^{2}\right), \quad \text { where } \quad \iota: \Gamma(\overline{\mathbf{K}} \otimes \overline{\mathbf{K}}) \subset \Gamma(\mathbf{L} \otimes \overline{\mathbf{K}})
$$

is the inclusion. Let $\mathcal{J}(\mathbf{L})$ denote the complex linear space of pairs $\left(Z, \phi d z^{2}\right)$ satisfying this equation.

Whenever a parametrized minimal surface $(f, \omega)$ has a nontrivial branch locus, the branch points give rise to nontrivial elements of $\mathcal{J}(\mathbf{L})$. Recall that 
the first Chern class of the line bundle $\mathbf{L}$ depends on the total branching order $\nu$; if $g$ is the genus of $\Sigma$ and $\nu$ is the total branching order,

$$
c_{1}(\mathbf{L})[\Sigma]=2-2 g+\nu .
$$

Lemma. The complex dimension of the space of tangential Jacobi fields is given by the formula:

$$
\operatorname{dim}_{\mathbb{C}} \mathcal{J}(\mathbf{L})= \begin{cases}3+\nu & \text { if } g=0 \\ 1+\nu & \text { if } g=1 \\ \nu & \text { if } g \geq 2\end{cases}
$$

This is an application of the Riemann-Roch theorem to the operator

$$
D^{\prime \prime}: \Gamma(\mathbf{L}) \rightarrow \Gamma\left(\mathbf{L} \otimes \mathbf{K}^{0,1}\right) .
$$

When the total branching order $\nu$ is zero, the cokernel of $D^{\prime \prime}$ is just the space of holomorphic quadratic differentials, and the only solutions to (34) are the holomorphic sections $\mathcal{O}\left(\mathbf{K}^{-\mathbf{1}}\right)$ of the holomorphic tangent bundle $\mathbf{K}^{-\mathbf{1}}$. This space of holomorphic sections is just the tangent space to the identity component $G$ of the group of conformal automorphisms, which is $\operatorname{PSL}(2, \mathbb{C})$ when $g=0$, $S^{1} \times S^{1}$ when $g=1$ and trivial when $g \geq 2$. Thus when $\nu=0$, $\operatorname{dim}_{\mathbb{C}} \mathcal{J}(\mathbf{L})$ is three, one or zero when the genus $g$ is zero, one or $\geq 2$ respectively.

Suppose next that $(f, \omega)$ is a conformal harmonic map with a single branch point $p$ of multiplicity one. Then $\mathbf{L}=\overline{\mathbf{K}} \otimes \zeta_{p}$, where $\zeta_{p}$ is the point bundle at $p$, and according to the Riemann-Roch theorem, the analytic index of $D^{\prime \prime}$ is given by the formula,

$$
\operatorname{dim} \mathcal{O}(\mathbf{L})-\operatorname{dim} \mathcal{O}\left(\mathbf{L}^{-1} \otimes \mathbf{K}\right)=1-g+c_{1}(\mathbf{L})[\Sigma] .
$$

Either the dimension of the kernel of $D^{\prime \prime}$ increases by one which yields a new element of $\mathcal{J}(\mathbf{L})$, or the dimension of the image of

$$
D^{\prime \prime}: \Gamma\left(\overline{\mathbf{K}} \otimes \zeta_{p}\right) \rightarrow \Gamma\left(\overline{\mathbf{K}} \otimes \overline{\mathbf{K}} \otimes \zeta_{p}\right)
$$

decreases by one. In the latter case, at least one nonzero antiholomorphic quadratic differential is covered by $D^{\prime \prime}$, which also gives a new element of $\mathcal{J}(\mathbf{L})$. (The space of new elements of $\mathcal{J}(\mathbf{L})$ obtained increasing $\nu$ by one is at most onedimensional, because given two such solutions, a nontrivial linear combination would vanish at $p$ and therefore lie within $\mathcal{J}(\overline{\mathbf{K}})$.)

The general case of the Lemma is proven by induction on $\nu$. Each time $\nu$ is increased by one, either a new holomorphic section of $\mathbf{L}$ is produced or a new antiholomorphic quadratic differential is covered by $D^{\prime \prime}$, in either case leading to an increase of one in the dimension of $\mathcal{J}(\mathbf{L})$.

Thus a minimal surface $(f, \omega)$ with total branching order $\nu$ always has a $\nu$ dimensional space of tangential Jacobi fields in addition to the space of Jacobi 
fields generated by the conformal automorphisms of $\Sigma$. If $(f, \omega)$ is a branched cover of a minimal surface without branch points, these Jacobi fields have a very natural interpretation; they form the tangent space to a critical submanifold of branched covers. It is clear that tangential Jacobi fields must play a key role in elucidating the geometry of prime minimal surfaces with nontrivial branch locus.

\section{References}

[1] E. D'Hoker, String theory in Quantum fields and strings: a course for mathematicians, Volume 2, American Mathematical Society, Providence RI, 1999.

[2] S. Donaldson and P. Kronheimer, The geometry of four-manifolds, Clarenden Press, Oxford, 1990.

[3] A. Fraser, Fundamental groups of manifolds with positive isotropic curvature, Ann. of Math. 158 (2003), 345-354.

[4] B. Lawson, Lectures on minimal submanifolds, Publish or Perish, Berkeley, 1980.

[5] D. McDuff and D. Salamon, J-holomorphic curves and symplectic topology, Amer. Math. Soc., Providence Rhode Island, 2004.

[6] M. Micallef, Stable minimal surfaces in Euclidean space, J. Differential Geometry 19 (1984), 57-84.

[7] M. Micallef and J. D. Moore, Minimal two-spheres and the topology of manifolds with positive curvature on totally isotropic two-planes, Annals of Math. 127 (1988), 199-227.

[8] M. Micallef and J. Wolfson, The second variation of area of minimal surfaces in four-manifolds, Math. Ann. 295 (1993), 245-267.

[9] R. Schoen and S. T. Yau, Lectures on harmonic maps, International Press, Boston, 1997.

[10] J. Simons, Minimal varieties in Riemannian manifolds, Annals of Math. 88 (1968), 62-105.

[11] S. Smale, An infinite-dimensional version of Sard's theorem, Amer. J. Math. 87 (1966), 861-866.

[12] B. White, The space of minimal submanifolds for varying Riemannian metrics Indiana Math. J. 40, (1991), 161-200. 